\title{
A METHOD OF GENERATING PART OF ARITHMETIC WITHOUT USE OF INTUITIVE LOGIC
}

\author{
BY W. V. QUINE*
}

1. Introduction. By an identity I mean an equation which is a theorem of ordinary arithmetic (and hence true for all values of its variables). What is meant here by "ordinary arithmetic" may be left to the reader, granted the following three reservations:

(a) The equations

$$
\begin{aligned}
x & =x-(y-y), \\
x-(y-z) & =z-(y-x),
\end{aligned}
$$

are identities, that is, theorems of ordinary arithmetic.

(b) Inference according to either of the following rules is valid in ordinary arithmetic:

(R) Substitute any one expression for all occurrences of any variable.

$\left(\mathrm{R}^{\prime}\right)$ Given $\alpha=\beta$, put $\alpha$ for $\beta$ anywhere.

(c) An equation whose members open with the same variable and contain only variables connected by subtraction, without parentheses, is not an identity unless each variable occurs exactly as many times in one member as in the other.

$A$ homogeneous linear identity with rational coefficients is an identity whose members contain only plus and minus signs, parentheses, " 0 ", variables, and rational numerical coefficients. Aside from the prefixture of such coefficients, no multiplicative juxtaposition is admissible. The expressions thus admitted can all be defined in terms of variables, parentheses and the signs of subtraction and equality. These definitions, which have the status of mere conventions of notational abbreviation, will be set forth in $\$ 3$.

It is the business of this paper to show that all homogeneous

* Society of Fellows, Harvard University. For suggestions in the preparation of this paper I am indebted to E. V. Huntington and T. P. Palmer. 
linear identities with rational coefficients, when thus analyzed into subtraction, equality, and variables, can be generated from (A) and (B) by means solely of mechanical substitution according to (R) and $\left(\mathrm{R}^{\prime}\right)$.

2. Generation of Theorems. (A) and (B) comprise the postulates, and $(R)$ and $\left(R^{\prime}\right)$ the rules of inference, of a calculus whose primitive language contains only variables, the functor of subtraction and the equality sign.* The parentheses represent nothing in the way of an additional primitive idea, but merely constitute a part of the notation of the functor of subtraction. This functor is the notational scheme of writing expressions in the respective blanks of the matrix " $-(\quad)$ ". No parentheses are needed enclosing the blank to the left of " - " in this matrix, for, as is easily verified relatively to subtraction or any other binary operation, the condition that all right-hand operands and only right-hands operands are to be parenthesized suffices in any context for the unique determination of the grouping of left-hand operands as well.

Strictly, (A) and (B) should appear as

$$
x=x-(y-(y)) \text { and } x-(y-(z))=z-(y-(x)) .
$$

The suppression of parentheses enclosing single letters is an ellipsis; in using $(R)$ and $\left(R^{\prime}\right)$, we are to imagine that single letters immediately to the right of minus signs are enclosed in parentheses, and we are to make such parentheses explicit when a complex is substituted for such a letter.

Let us proceed to the generation of theorems from (A) and (B) by $(R)$ and $\left(R^{\prime}\right)$. The manner of generation is indicated, to the right of each theorem, by literal or numerical reference to

* The primitive dyadic relation of equality might be suppressed in favor of a primitive predicate; if namely " $\mathfrak{B}$ " denote the predicate of equality with 0 , so that " $B \zeta$ " is read " $\zeta$ vanishes", we can render any equation $\alpha=\beta$ as $\mathfrak{B} \alpha-\beta$. (A) and (B) become

$\mathfrak{B} x-(x-(y-y))$ and $\mathfrak{B}(x-(y-z))-(z-(y-x))$. (R) remains unchanged, but $\left(\mathrm{R}^{\prime}\right)$ refers now to $\mathfrak{B} \alpha-\beta$ instead of $\alpha=\beta$.

Indeed, since in the thus modified calculus all theorems and postulates begin uniformly with "B̉", that letter might be dropped entirely; the list of theorems and postulates would then appear as a list of alternative expressions of 0 . A system is thus arrived at whose theorems and postulates are expressive of elements of the system itself, as is the case in the logical calculus of propositions. 
the postulates or intermediate theorems from which the theorem in question is derived. A single reference " $t$ " means that the generation proceeds by substitution in the theorem or postulate $(\mathrm{t})$ according to $(\mathrm{R})$. A double reference " $(s),(t)$ " means that the generation proceeds by replacement of an occurrence of the right member of (s), within $(t)$, by the left member of (s), on the authority of $\left(\mathrm{R}^{\prime}\right)$.

$$
\begin{aligned}
& x=x, \\
& \text { (A), (A). } \\
& x=x-(z-z) \text {, } \\
& \text { (A). } \\
& y-(x-z)=z-(x-y), \\
& y-(x-(z-z))=z-z-(x-y), \\
& \text { (5) } z-(z-z-(x-y))=x-y-(z-z-z) \text {, } \\
& \text { (6) } y-y-(x-x-x)=y-y-(x-x-x) \\
& \text { (21) } y-y-(x-x-x)=x \text {, } \\
& z-(y-x)=x-y-(z-z-z), \quad(7),(5) \text {. } \\
& x-(y-y)=y-y-(x-x-x) \text {, } \\
& x-y-z=w-w-(y-(x-z)), \quad(3),(9) \text {. } \\
& x-(y-z)=x-y-(z-z-z), \quad \text { (B), (10). } \\
& x-(y-y)=x-y-(y-y-y) \text {, } \\
& x-y-z=x-z-y, \\
& z-z-(x-y)=z-(x-y)-z, \\
& y-x=z-(x-y)-z, \\
& y-x=y-(x-z)-z, \\
& x=x-y-(y-y-y), \\
& x=y-y-(x-x-x), \quad(\mathrm{A}),(11) .
\end{aligned}
$$

3. Phrases. By a phrase I shall mean any expression built up of variables and the functor of subtraction. A more rigorous de- 
scription is the following: Letters, also the expression " $x-y$ ", are phrases, and if a letter in a phrase be replaced by a phrase (with restoration of requisite parentheses, in conformity with $\S 2)$ the result is a phrase. A phrase-equation is an equation whose members are phrases; a phrase-identity is a phrase-equation which is an identity.

A homogeneous linear identity with rational coefficients is not necessarily a phrase-identity, for, over and above expressions of subtraction, its members may involve expressions of algebraic negation or addition, the sign " 0 ", and rational numerical coefficients. These further devices are all definable, however, as means of abbreviating phrases or phrase-equations. " 0 " can be explained as an abbreviation for the specific expression " $x-x$ ". Negation can be accounted for by defining $-z$ in general as $0-z$, that is, by construing " $-(\quad)$ " as an abbreviation for " $0-(\quad)$ ". Addition can be introduced similarly by defining $x+y$ as $x-(-y)$, and the integral numerical coefficients are provided for by defining $2 x$ as $x+x, 3 x$ as $2 x+x$, and so on.* The symbols " $0 x$ " and " $1 x$ " may be taken as notational variants of " 0 " and " $x$ ".

Fractional numerical coefficients, finally, can be defined contextually by the following abbreviative conventions. An equation

$$
" \alpha-\beta-\cdots-\frac{m}{n} \zeta-\cdots-\theta=\omega ",
$$

with any disposition of parentheses, is an abbreviation for

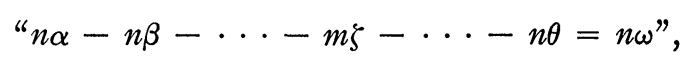

with the corresponding disposition of parentheses, where $m$ is any numeral and $n$ any numeral other than "0". The corresponding convention is adopted where the fraction occurs to the right of the equality sign. If several fractional coefficients occur in an equation, their explanation by the above conventions is to proceed in a left-to-right order. This set of conventions provides for the occurrence of fractional coefficients not only in subtraction but in the other contexts as well, when the

* See E. V. Huntington, Annals of Mathematics, (2), vol. 8 (1906), p. 15. 
definitions of the preceding paragraph are brought into play.*

Every homogeneous linear identity with rational coefficients is either a phrase-identity or an abbreviation of a phrase-identity by the above abbreviative conventions. The proof that every homogeneous linear identity with rational coefficients is, upon elimination of the above abbreviations, generable by $(\mathrm{R})$ and $\left(R^{\prime}\right)$ from (A) and (B), will therefore consist in showing that every phrase-identity is generable by $(R)$ and $\left(R^{\prime}\right)$ from (A) and (B).

4. Canonical Form of an Equation. An equation will be said to be of canonical form if each of its members is a phrase in which no parentheses occur and in which like letters appear consecutively, and if the initial letters of the two members are alike and the order of succession of unlike letters is, barring omissions, the same in both members. The canonical form is thus

$$
\begin{aligned}
v_{1}-v_{1}-v_{1}-\cdots-v_{2}-v_{2}-\cdots-v_{n}-v_{n} \\
\quad=v_{1}-v_{1}-v_{1}-\cdots-v_{2}-v_{2}-\cdots-v_{n}-v_{n}
\end{aligned}
$$

where $v_{i}$ and $v_{j}$ are unlike letters unless $i=j$, and where, for any $m$, the number of occurrences of " $-v_{m}$ " in either member may be 0 .

In saying that an equation $E$ is derivable from an equation $F$, I shall mean that $E$ is generable by (R) and $\left(\mathrm{R}^{\prime}\right)$ from (A), (B), and $F$. In saying that $E$ is interderivable with $F$, I shall mean that $E$ is derivable from $F$ and $F$ from $E$. In saying that an equation is reducible to canonical form, I shall mean that it is interderivable with an equation of canonical form.

Since the initial letters of the members of an equation of canonical form are alike, $\$ 1$ (c) tells us that an equation (i) of canonical form is not an identity unless each variable $v_{i}$ occurs the same number of times in one member as in the other. But under such circumstances the members of (i) are exact duplicates of each other, so that the equation is generable by $(R)$ from the principle (1) of self-equality. Since (1) is one of the

* It is seen from (A) and (B) that the structure of the calculus developed in $\$ 2$ admits of another application, where the elements are non-vanishing numbers and " $x-y$ " is re-interpreted as meaning " $x$ divided by $y$ ". Under this reinterpretation, 0 as above defined gives way to 1 , the negative gives way to the reciprocal, the sum to the product, and the numerical coefficients to the corresponding numerical exponents. 
theorems already generated by $(R)$ and $\left(R^{\prime}\right)$ from $(A)$ and (B), it follows that every identity of canonical form is generable by $(\mathrm{R})$ and $\left(\mathrm{R}^{\prime}\right)$ from $(\mathrm{A})$ and $(\mathrm{B})$.

By $\S 1(b)$, any equation generable by $(R)$ and $\left(R^{\prime}\right)$ from theorems of ordinary arithmetic is a theorem of ordinary arithmetic, and hence an identity. In view of $\$ 1(\mathrm{a})$, therefore, any equation generable by (R) and ( $\left.\mathrm{R}^{\prime}\right)$ from $(\mathrm{A}),(\mathrm{B})$, and $E$ is an identity if $E$ is an identity. Now let $E$ be an identity reducible to canonical form, that is, interderivable with some equation $F$ of canonical form. Equation $F$ is then an identity, since it is generable by $(\mathrm{R})$ and $\left(\mathrm{R}^{\prime}\right)$ from $(\mathrm{A}),(\mathrm{B})$, and $E$. But, being an identity of canonical form, $F$ is generable by $(\mathrm{R})$ and $\left(\mathrm{R}^{\prime}\right)$ from (A) and (B) alone. Hence $E$, derivable as it is from $F$, is likewise generable by $(R)$ and $\left(R^{\prime}\right)$ from $(A)$ and $(B)$. It is thus established that every identity $E$ which is reducible to canonical form is generable by $(R)$ and $\left(R^{\prime}\right)$ from $(A)$ and $(B)$.

In order to show then that every phrase-identity is generable by $(R)$ and $\left(R^{\prime}\right)$ from (A) and (B), it will be sufficient to prove that all phrase-equations (and hence in particular all phraseidentities) are reducible to canonical form. This proof occupies the remaining section.

5. Proof of Reducibility. Given any equation

$$
\alpha=\beta,
$$

the reverse equation (iv) is derivable as follows:

$$
\begin{aligned}
& \beta=\beta, \\
& \beta=\alpha,
\end{aligned}
$$

Hence the following principle is established.

I. Any equation $\alpha=\beta$ is interderivable with its reverse $\beta=\alpha$.

By the terms of a phrase $\alpha$ I mean all letters of $\alpha$ not lying within parentheses in $\alpha$, and all parenthesized phrases in $\alpha$ not lying within any broader parenthesis in $\alpha$. Since parentheses can open only to the right of a minus sign [see \$2], the initial term of a phrase is necessarily a letter.

From any phrase-equation

$$
x-\alpha_{1}-\alpha_{2}-\cdots-\alpha_{i}-\alpha_{i+1}-\cdots-\alpha_{n}=\omega,
$$

we can derive an equation (vii) as follows: 


$$
\begin{aligned}
& \text { (vi) } x-\alpha_{1}-\cdots-\alpha_{i-1}-\alpha_{i+1}-\alpha_{i} \\
& =x-\alpha_{1}-\cdots-\alpha_{i-1}-\alpha_{i}-\alpha_{i+1}, \\
& \text { (vii) } x-\alpha_{1}-\cdots-\alpha_{i-1}-\alpha_{i+1} \\
& -\alpha_{i}-\alpha_{i+2}-\cdots-\alpha_{n}=\omega,(\mathrm{vi}),(\mathrm{v}) .
\end{aligned}
$$

By the same process, with $\alpha_{i}$ and $\alpha_{i+1}$ interchanged, we can derive (v) from (vii). Thus any phrase-equation ( $v$ ) is interderivable with an equation (vii) resulting from the permutation of any non-initial term of the left member of (v) with its successor. Serial application of this principle establishes the interderivability of ( $\mathrm{v}$ ) with the equation resulting from any permutation of non-initial terms in the left member of (v). In view of I, furthermore, the same will be true relatively to the right member. Hence the following principle is inferred.

II. Any phrase-equation $E$ is interderivable with any equation resulting from permutation of non-initial terms within either member of $E$.

From any phrase-equation (viii) $y-\alpha_{1}-\alpha_{2}-\cdots-\alpha_{m}=x-\beta_{1}-\beta_{2}-\cdots-\beta_{n}$, we can derive an equation (ix) as follows:

$$
\begin{aligned}
y & -\alpha_{1}-\cdots-\alpha_{m} \\
& =y-y-(x-x-x)-\beta_{1}-\cdots-\beta_{n}, \quad \text { (21), (viii). }
\end{aligned}
$$

Conversely, (viii) is derivable from (ix) as follows:

[viii] $y-\alpha_{1}-\cdots-\alpha_{m}=x-\beta_{1}-\cdots-\beta_{n}, \quad$ (20), (ix).

Therefore (viii) is interderivable with (ix). Hence the following principle is established.

III. Any phrase-equation is interderivable with a phraseequation, the initial letters of whose members are alike.

Since parentheses can occur in a phrase only by way of enclosing a non-initial term thereof, it follows from II that if any parenthesized expression in a phrase-equation be transported to the end of the member in which it occurs, the resulting equation will be interderivable with the original one. Thus, in particular, any phrase-equation 
(x) $\quad x-\alpha_{1}-\alpha_{2}-\cdots-\alpha_{m}$

$$
=x-\beta_{1}-\beta_{2}-\cdots-\beta_{i-1}-(\zeta-\theta)-\beta_{i+1}-\cdots-\beta_{n},
$$

whose members have like initial letters and whose right member contains parentheses as indicated, is interderivable with

(xi) $\quad x-\alpha_{1}-\cdots-\alpha_{m}$

$$
=x-\beta_{1}-\cdots-\beta_{i-1}-\beta_{i+1}-\cdots-\beta_{n}-(\zeta-\theta) .
$$

Now from (xi) we can derive an equation (xiii) as follows:

$$
\begin{aligned}
& \text { (xii) } x-\beta_{1}-\cdots-\beta_{i-1}-\beta_{i+1}-\cdots-\beta_{n}-\zeta \\
& =x-\beta_{1}-\cdots-\beta_{i-1}-\beta_{i+1}-\cdots-\beta_{n} \\
& -(\zeta-\theta)-\theta \text {, } \\
& =x-\alpha_{1}-\cdots-\alpha_{m}-\theta \text {, }
\end{aligned}
$$

Conversely, (xi) is derivable from (xiii) as follows:

$$
\begin{aligned}
& \text { (xiv) } x-\alpha_{1}-\cdots-\alpha_{m}=x-\alpha_{1}-\cdots-\alpha_{m} \\
& -\theta-(\theta-\theta-\theta) \text {, } \\
& \text { (xv) } x-\alpha_{1}-\cdots-\alpha_{m}=x-\beta_{1}-\cdots-\beta_{i-1}-\beta_{i+1} \\
& -\cdots-\beta_{n}-\zeta-(\theta-\theta-\theta), \quad \text { (xiii), (xiv), } \\
& \text { (xvi) } x-\beta_{1}-\cdots-\beta_{i-1}-\beta_{i+1}-\cdots-\beta_{n}-(\zeta-\theta) \\
& =x-\beta_{1}-\cdots-\beta_{i-1}-\beta_{i+1}-\cdots-\beta_{n} \\
& -\zeta-(\theta-\theta-\theta) \text {, } \\
& -\beta_{i+1}-\cdots-\beta_{n}-(\zeta-\theta), \quad \text { (xvi), (xv). }
\end{aligned}
$$

Thus (xi), and hence also (x), is interderivable with (xiii). By I, then, the reverse of $(\mathrm{x})$ is likewise interderivable with (xiii). But in the reverse of $(x),(\zeta-\theta)$ occurs in the left member rather than the right. Hence, in conclusion, a phrase-equation whose members have initial letters and either of whose members contains a parenthesized expression, is interderivable with a phrase-equation (xiii) in which the members still have like initial letters but in which the parenthesized expression is broken up. Serial application of this principle establishes the following one. 
IV. Any phrase-equation whose members have like initial letters is interderivable with a phrase-equation whose members are free of parentheses and have like initial letters.

From the definition of the "terms" of a phrase, it is clear that if a phrase is free of parentheses all its terms are letters. Hence, by II, any phrase-equation $E$ whose members are free of parentheses is interderivable with any phrase-equation resulting from permutation of non-initial letters within members of $E$. Therefore any phrase-equation whose members are free of parentheses and have like initial letters is interderivable with an equation of canonical form. It then follows, by III and IV, that every phrase-equation is reducible to canonical form.

In view of $\S \S 3-4$, this concludes the proof that upon elimination of abbreviations all homogeneous linear identities with rational coefficients are generable by $(R)$ and $\left(R^{\prime}\right)$ from (A) and (B).

HARVARD UNIVERSITY

\section{A CHARACTERISTIC PROPERTY OF SURFACES OF NEGATIVE CURVATURE $\dagger$}

\section{BY E. F. BECKENBACH}

1. Introduction. Let there be given a piece of surface $S$ in a representation

(1) $S: \quad x=x(u, v), y=y(u, v), z=z(u, v), u^{2}+v^{2}<\rho^{2}$, with the following properties.

(a) $x(u, v), y(u, v), z(u, v)$ have continuous partial derivatives of the third order.

(b) The representation is isothermic; that is to say, $E=G$, $F=0$, where

$E=x_{u}{ }^{2}+y_{u}{ }^{2}+z_{u}{ }^{2}, F=x_{u} x_{v}+y_{u} y_{v}+z_{u} z_{v}, G=x_{v}{ }^{2}+y_{v}{ }^{2}+z_{v}{ }^{2}$,

the subscripts denoting differentiation.

We put $E=G=\lambda(u, v)$. Then $\lambda(u, v) \geqq 0$, and the representation is conformal except at points where $\lambda(u, v)=0$. The cus-

$\dagger$ Presented to the Society, April 7, 1934. 